two-ring machine optimized for 100 $\mathrm{GeV}$ per beam. It was then decided to study carefully a single ring LEP optimized for $70 \mathrm{GeV}$ per beam, with a radius of $3.5 \mathrm{~km}$.

This second proposal, published in August 1978, was costed out at about $100 \mathrm{M} \mathrm{SwFr}$, i.e. about half the original sum, and construction was estimated to take 5-7 years. It envisaged a machine built in a tunnel of $4 \mathrm{~m}$ diameter cross-section, with eight straight sections, each of which was an interaction region. Four bunches each of electrons and positrons travelled in opposite directions and luminosity in the interaction regions was to be $10^{32} \mathrm{~cm}^{-2} \mathrm{~s}^{-1}$. This proposal was based on the use of conventional iron-cored magnets but it was anticipated that later these could be replaced by superconducting magnets in order to achieve $100+100 \mathrm{GeV}$. It can be noted that the cost is only a little greater than that of the SPS, and LEP could be built inside the CERN budget if this was maintained at its present level by shutting down the $33+33 \mathrm{GeV}$ pp collider and the $600 \mathrm{MeV}$ synchrocyclotron and taking other austerity measures.

When the physics community had examined the new proposal, it pressed for the energy attainable with conventional techniques to be raised to 85 $+85 \mathrm{GeV}$ and CERN was once more asked to review its design. "Version 8 " is now under study and due to be presented in time for ECFA to make recommendations on energy, radius and site by the end of the year. Major ring circumference has been increased to $30607 \mathrm{~m}$ and studies are being made on how costs can be held down still more. One technique that is being investigated, for example, is the use of concrete magnets and although superconducting magnets are considered too advanced for this generation it can be anticipated that superconducting RF cavities would be introduced. Such studies are being made in close collaboration with all the main western Europe high energy physics laboratories. PETRA, for example will be used for tests on beam dynamics; Orsay is specializing in the injector and so on.

It is also proposed to build the machine in steps which would allow costs to be spread. First step would be $50+50 \mathrm{GeV}$ at maximum luminosity, second $72+72$ and third $80 / 85$ $+80 / 85$. First operation could begin in 1988.

It was noted that the synchrotron radiation losses at these energies would be respectively $5.76 \mathrm{MW}, 13 \mathrm{MW}$ and 20-24 MW per beam and a call has gone out to see whether at least a part of this radiation could not be put to good use.

Parallel to the machine studies led dy J. B. Adams, ECFA has set ap two working groups. One under, A. Zichichi is studying the experimental programme that might be associated with the machine and involves more than 360 physicists and engineers from all over western Europe. The second, chaired by $\mathrm{J}$. Mulvey is looking at the consequences of such a project on subnuclear physics research in the CERN Member States. Abdus Salam's conclusions on the LEP project were that never before had there been a machine out of which you could be sure to get so much physics.

\section{Machines Around the World}

It is interesting to see how the several continents have become identified with different projects and one is seeing a world programme emerging where each major region specializes in something different.

China it seems, is concentrating for the present on a generously dimensioned $50 \mathrm{GeV}$ proton synchrotron. At NAL Batavia in the USA, a second ring with superconducting megnets is being installed in the present 500 $\mathrm{GeV}$ annulus which will be used, to begin with as a proton synchrotron of $1 \mathrm{TeV}$ (the Tevatron). The presence of two rings then gives opportunities for different configurations in the future and proposals have been made for pp: $1000+250 \mathrm{GeV}$ and pp: 250 $+250 \mathrm{GeV}$ or with cooling 1000 $+1000 \mathrm{GeV}$. The USSR multi-TeV fixed target proton synchrotron project UNK, with provision for later $\mathrm{pp}$ work at $2.2 \mathrm{TeV}$ and then $6 \mathrm{TeV}$ centre of mass energy is described in the preceding contribution. Other hadron colliders are: Isabelle in the US pp: $400+$ $400 \mathrm{GeV}$; the CERN modified SPS facility for $p p$ with $500 \mathrm{GeV}$ in the centre of mass; Tristan in Japan with pp and $400 \mathrm{GeV}$ in the centre of mass. LEP with $\mathrm{e}^{+} \mathrm{e}^{-}$near $100+100 \mathrm{GeV}$ is for the moment on its own. At Novosibirsk, however, a new approach is being considered, namely an $\mathrm{e}^{+} \mathrm{e}^{-}$ one shot linear machine which might be built in two stages, if the problems of accelerating very rapidly, intense and small phase bunches could be solved. First stage could be a $1 \mathrm{~km}$ long pair to give $100+100 \mathrm{GeV}$ followed by a second step of three times the length and energy. Repetition frequency might be $10 \mathrm{~Hz}$.

High energy physics it is clear is no longer following a single route and the diversification of machines is meaning that a world machine is giving place in physicists' minds to a world programme in which all the big laboratories are complementary and international in the widest sense.

\title{
European Synchrotron Radiation Facility
}

A summary of his lecture prepared by Y. Farge, of Orsay, chairman of the ad hoc committee set up by the European Science Foundation to study European synchrotron radiation requirements.

In 1976, the European Science Foundation, located in Strasbourg, set up a study group on synchrotron radiation under the chairmanship of H. Maier-Leibnitz. This group was composed of sixteen scientists, mainly from various synchrotron radiation facilities in western Europe which comprise: Bonn (Fed. Rep. of Germany), Daresbury (United Kingdom), Desy (Fed. Rep. of Germany), Frascati (Italy), Lund (Sweden), Orsay (Sweden). It presented the following recommendations and conclusions.

1. There will be a large discrepancy between the number of scientists who wish to use synchrotron radiation and the number of stations available on existing or proposed machines.
2. Existing high energy physics machines in general are not well adapted for synchrotron radiation work.

3. Energy consumption of some existing storage rings make their operation for synchrotron radiation work a very expensive proposition.

4. The design of many existing machines precludes the use of wigglers.

5. Strong efforts should be made to obtain dedicated beam time at existing high energy physics storage rings. Additional beam lines should be installed at these facilities to exploit them in an optimised way for synchrotron radiation research.

6. Small storage rings can be realised on a national scale. 
7. In every synchrotron radiation facility, provision should be made to accommodate researchers from other countries. A special fund should be made available in order to promote intra-European mobility.

8. A large effort to build a dedicated hard X-ray storage ring and appropriate advanced instrumentation with a design which goes beyond that of present day projects is recommended.

9. This machine should be operational by about 1985 . Its construction should be started in 1980 .

10. An interdisciplinary working group should be constituted with the task of studying the parameters of this European X-ray synchrotron radiation laboratory, its cost and its organisation. The laboratroy should approach the final state of the art.

As a consequence of this, an ad hoc committee was set up in October 1977 by the ESF, with two goals in view:

- to improve relations between existing centres;

- to make a feasibility study of a large $\mathrm{X}$-ray synchrotron radiation source.

The committee, chaired by Y. Farge, is now working on these problems, and this paper reports on the progress made concerning the study of the X-ray machine. It should however, be noted that work on this project is not yet finished and the final report will be presented to the ESF General Assembly in November 1979. Nevertheless, it is possible to give some indications on the work accomplished and the general form of the machine.

In order to organise the work, two sub-groups were established. One of these, chaired by D. J. Thompson, from Daresbury Laboratory, has in the region of sixteen members from all the high energy physics laboratories in western Europe, and it studies the details of the machine. The "Instruments and Beamlines" sub-group, chaired by B. Furas, from Copenhagen, has approximately twelve members, mainly from existing synchrotron radiation facilities, and it aims to distinguish the best instruments for such an excellent X-ray machine. However, the most important part of the work is clearly the Scientific Case for such a facility. Due to the very large number of scientific fields involved in synchrotron radiation (from atomic physics to biology and applied sciences) it was not possible to create a special sub-group, and the committee has asked twenty or so of our European colleagues to think along the lines of the use of such a machine in many different disciplines; this work has been performed either by small meetings in regular international conferences or by specialist meetings organised for this purpose.

\section{The Scientific Case}

The Scientific Case is not yet complete and it is only possible to give some general indications of what could be done with a very powerful $X$-ray source that cannot be done with existing storage rings:

\section{Spectroscopic Studies}

Deep atomic level studies by photoelectron spectroscopy; ESCA studies with tunable excitation wavelengths up to $50 \mathrm{keV}$; Mössbauer spectroscopy with nuclear transitions of up to 200 keV ; Compton scattering measurement with tunable excitation of up to $400 \mathrm{keV}$; very fast EXAFS measurements (approx. 1/s); soft X-ray photoemission and detailed study of surfaces (magnetism for example).

\section{Elastic Scattering}

It is essentially the intensity gain which is interesting with a very powerful X-ray machine for X-ray topography, structure determinations, small angle X-ray scattering, X-ray diffuse scattering, etc... Actual rings are producing tunable $\mathrm{X}$-rays, with a gain in exposure times of between 50 and $10^{4}$ depending on the geometry of the experiments. It is possible, with these machines, to reach structural information with typical times larger or equal to $10 \mathrm{~ms}$. With a more powerful ring, it would be of great importance to reach $1 \mu \mathrm{s}$, a typical time which is very promising for many studies in material sciences (phase transitions, polymers, metallurgy, solid state chemistry, etc...) but also in biology where time dependent structural information is so important.

\section{Applied Sciences}

The gain in intensity should permit the performance of fast X-ray and soft $X$-ray imaging as well as fast $X$-ray holography. It would also be possible to measure accurately, traces of elements with their chemical environment.

\section{The Machine}

It is quite clear that many new scientific fields can be approched if it is possible to gain two or three orders of magnitude in X-ray intensity. The machine sub-group has defined a machine with the following characteristics:

- critical wavelength: $\lambda_{c}=1 \AA$ in normal bending magnets

(useful wavelengths down to $\lambda_{c} / 6$ ); - wigglers in straight section with: i. $c=0.25 \AA$

- very good focusing of the electron beam in bending magnets and wigglers;

- undulators on long straight sections with tunable wavelength emission $(1 \AA<\lambda<100 \AA)$ and very high brightness.

This design is a very good one compared to existing machines or X-ray machines under construction anywhere in the world. The brightness, defined as the photon flux in a given solid angle and a given band pass, divided by the source area, will be five times better than the one at the facility planned at Brookhaven and 50 to 200 times better than at existing machines.

The brightness in undulators will be $10^{3}$ higher in normal bending magnets, though it must be remembered that all the radiation (in a given narrow bandpass) is emitted in a very small solid angle and the number of photons of a given wavelength is therefore not increased by increasing the acceptance of the beam line. It is quite clear that such a machine would constitute a great improvement with respect to other machines. It would be very helpful to build it during the 1980's in order to keep the very good quality which exists in Europe at present in the field of synchrotron radiation, but also to use such a facility as a centre where European scientists from different countries and different fields could work together.

\section{European Synchrotron Radiation Facility}

$\begin{array}{ll}\text { Energy } & 5 \mathrm{GeV} \\ \text { Beam } & 565 \mathrm{~mA} \\ \text { Circumference } & 604 \mathrm{~m} \\ \text { Radius of bending magnets } & 22.36 \mathrm{~m} \\ \text { Critical wavelength in bending magnets } & 1 \AA \\ \text { Critical wavelength in wigglers } & 0.25 \AA \\ \text { Photon flux at } 1 \AA \text { (per m rad horiz.; } 0.1 \% \text { band width) } & 4.5 \times 10^{13} \mathrm{ph} / \mathrm{s} \\ & \end{array}$

Beam size at the middle of:

$$
\text { - Dipole magnets }
$$

- $6.8 \mathrm{~m}$ straight sections

- $3 \mathrm{~m}$ straight sections

Total synchrotron radiation

power with 6 wigglers

Number of bending magnets

Number of undulators

$\begin{array}{ll}\sigma_{x}, \mathrm{~mm} & \sigma_{z} \mathrm{~mm} \\ 0.18 & 0.11 \\ 0.50 & 0.07 \\ 0.11 & 0.03\end{array}$

$1.5 \mathrm{MW}$

48 